\title{
Potentialities of LL37 for Wound Healing Applications: Study of Its Activity in Synergy with Biodegradable Composites Made of PVA and CA
}

\author{
Helena P. Felgueiras ${ }^{(\bowtie)}$, Marta A. Teixeira, \\ and M. Teresa P. Amorim \\ Centre for Textile Science and Technology, University of Minho, \\ Campus de Azurém, 4800-058 Guimarães, Portugal \\ helena.felgueiras@2c2t.uminho.pt
}

\begin{abstract}
Wound healing is a dynamic and complex process that results from the interaction between cytokines, growth factors, blood components and the extracellular matrix. Conventional dressings made of natural or synthetic materials have only the ability to manage the wound and protect it from repeated trauma. With the advancement of technology, wound dressings have evolved and are now capable of intervening in the healing process by targeting specific features of the wound, aside from protecting the wounded site. In this work, bioactive dressings capable of promoting healing and fighting infection in chronic wounds were explored. Various antimicrobial biomolecules were examined in light of their pathogen fighting skills and immunoregulatory potentialities. Dressing production processes were also investigated. Biodegradable composite dressings made of poly(vinyl alcohol), polycaprolactone, chitosan and cellulose blends were our main focus. The goal was to evaluate the synergistic effect of biomolecules and biodegradable polymeric dressings, considering the local and systemic treatment demands of chronic wounds.
\end{abstract}

Keywords: Biodegradable polymers - Active biomolecules ·

Antimicrobial agents $\cdot$ Bioactive dressings $\cdot$ Effective chronic wound healing

\section{Introduction}

Wound healing is a systemic process that requires the interaction of a complex cascade of cellular events that generate resurfacing, reconstitution and restoration of the tensile strength of the injured skin, establishing a provisional tissue from which a complete regenerated epidermis is built. Skin regeneration in acute wounds evolves by following the phases of healing (coagulation, inflammation, proliferation and remodeling), in a predictable amount of time. To the contrary, chronic wounds can stall in one phase of healing (i.e. inflammation), developing a defective cell matrix colonized by bacteria (biofilms) and characterized by moisture imbalance, that requires much more time to heal $[1,2]$. In light of these issues, new generation of wound dressings have been proposed, and engineered aiming a more active intervention in the healing process that surpasses the simple protection/management of conventional, passive dressings. 
There is a growing interest in the development of biodegradable dressings from composites made of natural and synthetic biopolymers. Natural polymers arise from renewable resources, being highly desirable in wound healing for their biocompatibility and non-toxic nature. On its turn, biodegradable synthetic polymers have shown exceptional mechanical features that when combined with natural resources provide structural integrity $[2,3]$. Bioactive dressings are a fairly recent class of wound dressings responsible for promoting wound healing via the incorporation of biomolecules of interest, including antimicrobial peptides (AMPs), proteins, growth factors, ions, plant extracts, etc. [4, 5]. In the present work, we propose to evaluate the impact of bioactive dressings made of combinations of poly(vinyl alcohol) (PVA), polycaprolactone (PCL), chitosan (CS) and cellulose, modified with active biomolecules, for the prospective treatment of chronic wounds.

\subsection{Bioactive Dressings and the Incorporated Biomolecules}

Bioactive dressings are made of biodegradable, non-toxic biomaterials that play an active role in the normal healing of wounds and new tissue formation. They are based on the principle of creating and maintaining a moist wound environment that prevents the formation of a scab by facilitating exudates absorbency, oxygen exchange and controlling the $\mathrm{pH}$ on site. Bioactive dressings usually exhibit a fibrous structure in which are incorporated active compounds for site delivery (Fig. 1). These biomolecules intervene in the wound healing either directly or indirectly as cleansing or debridement agents removing necrotic tissues, as antimicrobials fighting infections, or as growth agents instigating tissue regeneration. Currently, the biomolecules considered most important are the AMPs, proteins and growth factors $[4,5]$.
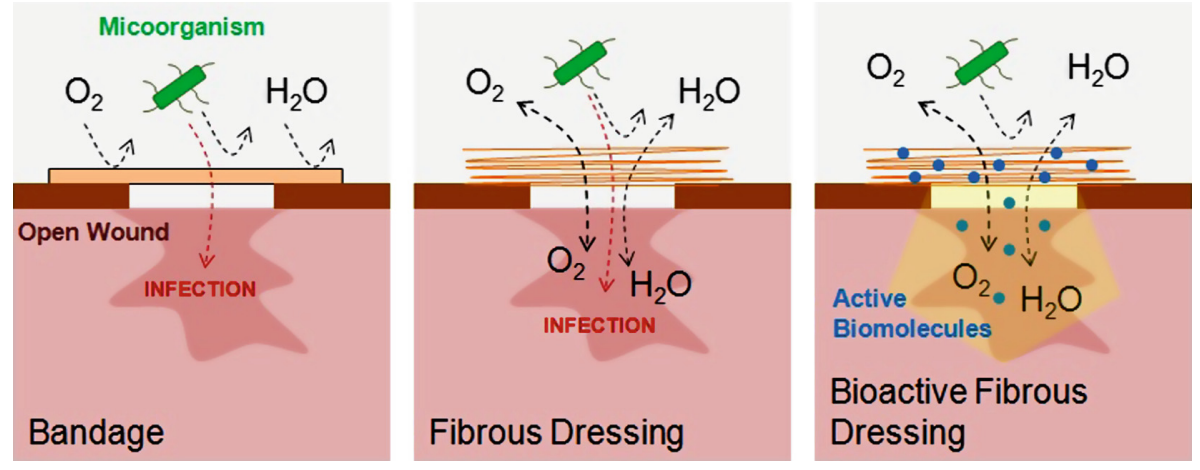

Fig. 1. Bioactive fibrous dressings impregnated with active biomolecules capable of fighting infection and promoting wound healing.

AMPs form an integral part of our innate immune system, being capable of killing pathogens in few seconds and, thus, reducing the likelihood of these microorganisms to develop resistance. They are low molecular weight molecules formed of 5-100 amino acids, with cationic charge. AMPs are usually classified in terms of their target 
microorganism, being the bacterial AMPs the most commonly employed in chronic wound healing. Because of their effective nature and natural-origin, the AMPs LL37, the only human cathelicidin-derived AMP, the pexiganan and the cecropin are the most researched; these AMPs prevent and/or treat infections and can even aid in the healing process $[2,6]$. Regarding proteins, collagen is without match the most important extracellular matrix (ECM) element for wound healing. Collagen exhibits excellent biocompatibility and has been highlighted as uniquely suited for wound healing because of its intervention in all phases of wound healing: promotes wound debridement, stimulates the clotting cascade, acts as a sacrificial substrate for proteolytic enzymes, and instigates tissue remodeling [3]. Finally, the growth factors, which take an active physiological role in chronic wound healing, have been shown to intervene in the stimulation of angiogenesis and cell proliferation, which affect both the production and degradation of the ECM, and in the inflammatory and remodeling phases. A large group of growth factors have been reported to participate in the healing process, including the epidermal growth factor (EGF), the platelet derived growth factor (PDGF), the fibroblast growth factor (FGF) and the transforming growth factor (TGF), to name a few [5].

\subsection{Composites Functionalized with Biomolecules for Wound Healing}

Bioactive dressings based on natural-origin materials, such as collagen and CS, have been reported to accelerate granulation tissue formation and epithelialization above synthetic dressings. However, their hydrophilicity, which difficults the control of antibiotic release, and their low tensile strength resistance have been pointed as disadvantages [7]. Electrospun PCL and PVA mats are suitable to treat acute and chronic wounds as they mimic the ECM structure. However, their use is limited by their poor antimicrobial properties. Hence, natural and synthetic polymeric combinations are desirable. PCL-collagen composites have been established as excellent triggers for integrin- $\beta 1$ signaling pathways to regulate the growth of fibroblasts and initiate wound healing [8]. Also, they have both been used in the delivery of EGF factors. CS has been employed in the delivery of EGF as well, for the treatment of full thickness wounds [9]. Coaxial electrospun PCL/PVA-chitosan nanofibrous scaffolds have been engineered for drug and gene delivery $[8,10]$. Bioengineered cellulose can be used as scaffolding material for chronic wound dressings, by reducing pain and shortening healing time. Just recently, we have uncovered that porous, flexible mats made of PVA and cellulose blends, processed to resemble actual skin, when functionalized with the AMP LL37 can stimulate healing and decrease microbial action in more than $80 \%$. Preliminary data on the immobilization of this AMP and pexiganan on PVA/cellulose acetate (CA) and PVA/PCL mats has revealed an accelerated clotting time and bactericidal effect against both Gram-positive and Gram-negative bacteria [unpublished work]. Pexiganan-incorporated collagen matrices have also been studied with successful results in infected wounds [8]. The use of functionalized AMPs in wound healing is still quite recent. However, new formulations are being developed everyday and are expected to overcome quickly the limitations of the conventional strategies. 


\section{Conclusions and Future Work}

The role of bioactive dressings is to deliver active biomolecules to the wounded site, to promote healing while fighting infections. At the present, we are studying combinations of PVA/CA, PVA/PCL and PVA/CS impregnated with immunoregulatory peptides and collagen for chronic wound healing applications. Using phase-inversion and electrospinning techniques, flexible, porous films and reticulated networks are being fashioned with properties that mimic the actual skin. In a near future, we intend to examine the synergistic performance of biomolecules by combining different AMPs, with different action modes, in a single formulation. We are confident these new systems aside from being target-effective against pathogens will also contribute effectively to the healing process by accelerating it.

Acknowledgements. Authors acknowledge the Portuguese Foundation for Science and Technology (FCT), FEDER funds by means of Competitive Factors Operational Program (POCI) for funding the projects POCI-01-0145-FEDER-028074 and UID/CTM/00264/2019.

\section{References}

1. Martin, P., Nunan, R.: Cellular and molecular mechanisms of repair in acute and chronic wound healing. Br. J. Dermatol. 173, 370-378 (2015)

2. Felgueiras, H.P., Amorim, M.T.P.: Functionalization of electrospun polymeric wound dressings with antimicrobial peptides. Colloids Surf. B 156, 133-148 (2017)

3. Felgueiras, H.P., Amorim, M.T.P.: Electrospun polymeric dressings functionalized with antimicrobial peptides and collagen type I for enhanced wound healing. In: 17th World Textile Conference, AUTEX 2017. IOP Conference Series: Materials Science and Engineering, vol. 254, p. 062004 (2017)

4. Georgescu, M., Chifiriu, M.C., Marutescu, L., Gheorghe, I., Lazar, V., Bolocan, A., Bertesteanu, S.: Bioactive wound dressings for the management of chronic wounds. Curr. Org. Chem. 21(1), 53-63 (2017)

5. Boateng, J.S., Matthews, K.H., Stevens, H.N.E., Eccleston, G.M.: Wound healing dressings and drug delivery systems: a review. J. Pharm. Sci. 97(8), 2892-2923 (2008)

6. Querido, M.M., Felgueiras, H.P., Rai, A., Costa, F., Monteiro, C., Borges, I., Oliveira, D., Ferreira, L., Martins, M.C.L.: Cecropin-melittin functionalized polyurethane surfaces prevent Staphylococcus epidermidis adhesion without inducing platelet adhesion and activation. Adv. Mater. Interfaces 5, 1801390 (2018)

7. Mogoşanu, G.D., Grumezescu, A.M.: Natural and synthetic polymers for wounds and burns dressing. Int. J. Pharm. 463(2), 127-136 (2014)

8. Mir, M., Ali, M.N., Barakullah, A., Gulzar, A., Arshad, M., Fatima, S., Asad, M.: Synthetic polymeric biomaterials for wound healing: a review. Prog. Biomater. 7, 1-21 (2018)

9. Park, J.W., Hwang, S.R., Yoon, I.-S.: Advanced growth factor delivery systems in wound management and skin regeneration. Molecules 22(8), 1259-1279 (2017)

10. Silva, I.O., Ladchumananandasivam, R., Nascimento, J.H.O., Silva, K.O.S., Oliveira, F.R., Souto, A.P., Felgueiras, H.P., Zille, A.: Multifunctional chitosan/gold nanoparticles synergistic coatings for biomedical textiles. Nanomaterials 9(8), 1064-1085 (2019). https://doi.org/10.3390/nano9081064 